\title{
M3 Muscarinic Receptor Signaling Stabilizes a Novel Mutant Human Ether-a-Go-Go-Related Gene Channel Protein via Phosphorylation of Heat Shock Factor 1 in Transfected Cells
}

\author{
Endang Mahati, MD; Peili Li, MD, PhD; Yasutaka Kurata, MD, PhD; \\ Nani Maharani, MD, PhD; Nobuhito Ikeda, PhD; Shinji Sakata, MD, PhD; \\ Kazuyoshi Ogura, MD, PhD; Junichiro Miake, MD, PhD; Takeshi Aiba, MD, PhD; \\ Wataru Shimizu, MD, PhD; Naoe Nakasone; Haruaki Ninomiya, MD, PhD; \\ Katsumi Higaki, MD, PhD; Kazuhiro Yamamoto, MD, PhD; Akira Nakai, MD, PhD; \\ Yasuaki Shirayoshi, PhD; Ichiro Hisatome, $\mathrm{MD}, \mathrm{PhD}$
}

\begin{abstract}
Background: Long QT syndrome 2 (LQT2) is caused by mutations in the human ether-a-go-go-related gene ( $h E R G$ ). Most of its mutations give rise to unstable hERG proteins degraded by the proteasome. Recently, carbachol was reported to stabilize the wild-type $h E R G$-FLAG via activation of the muscarinic type 3 receptor (M3-mAChR). Its action on mutant hERG-FLAG, however, remains uninvestigated.
\end{abstract}

\begin{abstract}
Methods and Results: A novel mutant hERG-FLAG carried 2 mutations: an amino acid substitution G572S and an in-frame insertion D1037_V1038insGD. When expressed in HEK293 cells, this mutant hERG-FLAG was degraded by the proteasome and failed to be transported to the cell surface. Carbachol restored stability of the mutant hERGFLAG and facilitated cell-surface expression. Carbachol activated PKC, augmented phosphorylation of heat shock factor 1 (HSF1) and enhanced expression of heat shock proteins (hsps), hsp70 and hsp90. Both a M3-mAChR antagonist, 4-DAMP, and a PKC inhibitor, bisindolylmaleimide, abolished carbachol-induced stabilization of the mutant hERG-FLAG.
\end{abstract}

Conclusions: M3-mAChR activation leads to enhancement of hsp expression via PKC-dependent phosphorylation of HSF1, thereby stabilizing the mutant hERG-FLAG protein. Thus, M3-mAChR activators may have a therapeutic value for patients with LQT2. (Circ J 2016; 80: 2443-2452)

Key Words: Carbachol; Heat shock factor 1 (HSF1); Human ether-a-go-go-related gene (hERG); Long QT syndrome type 2 (LQT2); M3-mACh

Received July 19, 2016; revised manuscript received September 28, 2016; accepted October 13, 2016; released online November 1, 2016 Time for primary review: 16 days

Division of Regenerative Medicine and Therapeutics, Department of Genetic Medicine and Regenerative Therapeutics, Institute of Regenerative Medicine and Biofunction, Tottori University Graduate School of Medical Science, Yonago (E.M., P.L., N.M., N.I., Y.S., I.H.), Japan; Department of Pharmacology and Therapeutics (E.M., N.M.), Center for Biomedical Research (CEBIOR) (N.M.), Faculty of Medicine, Diponegoro University, Semarang, Indonesia; Department of Physiology, Kanazawa Medical University, Kanazawa (Y.K.); Department of Pediatrics, Faculty of Medicine (S.S.), Division of Cardiovascular Medicine, Department of Molecular Medicine and Therapeutics, Faculty of Medicine (K.O., J.M., K.Y.), Department of Biological Regulation, Faculty of Medicine (N.N., H.N.), Division of Functional Genomics, Research Center for Bioscience and Technology (K.H.), Tottori University, Yonago; Department of Cardiovascular Medicine, National Cerebral and Cardiovascular Center, Suita (T.A.); Division of Cardiology and Regenerative Medicine, Nippon Medical School, Tokyo (W.S.); and Department of Biochemistry and Molecular Biology, Yamaguchi University School of Medicine, Ube (A.N.), Japan

The first author, E.M., along with the present study, has been selected as a finalist for the Young Investigator's Award for International students in 2016 JCS.

Mailing address: Li Peili, MD, PhD, Division of Regenerative Medicine and Therapeutics, Department of Genetic Medicine and Regenerative Therapeutics, Institute of Regenerative Medicine and Biofunction, Tottori University Graduate School of Medical Science, 86 Nishi-cho, Yonago 683-8503, Japan. E-mail: peili-li@med.tottori-u.ac.jp

ISSN-1346-9843 doi:10.1253/circj.CJ-16-0712

All rights are reserved to the Japanese Circulation Society. For permissions, please e-mail: cj@j-circ.or.jp 
$\boldsymbol{K}$ $\mathrm{CNH} 2$ encodes the $\alpha$ subunit of the human ether-a-gogo-related gene $(h E R G)$ channel that elicits the rapidly activating delayed-rectifier potassium current $\left(I_{\mathrm{Kr}}\right)$ responsible for action potential (AP) repolarization. ${ }^{1}$ Its mutation causes long QT syndrome type 2 (LQT2), which is characterized by prolongation of the QTc interval in electrocardiogram, and a high incidence of lethal arrhythmias. ${ }^{2,3}$ Most of the mutant hERG-FLAG have trafficking defects that result in reduced protein expression on the plasma membrane (PM). ${ }^{4}$

Heat shock factor 1 (HSF1) is a transcription factor that regulates expression of heat shock proteins (hsps). ${ }^{5}$ Hsps play a pivotal role in maturation and assemble of hERG channels. ${ }^{6}$ It has been reported that both hsp70 and hsp90 could facilitate maturation of the wild-type (WT) hERG-FLAG, and that hsp70 could facilitate that of mutant hERG-FLAG as well., ${ }^{7,8}$

The parasympathetic nervous system exerts negative inotropic and chronotropic actions on the heart through activation of muscarinic acetylcholine receptors (mAChRs). Among the five mAChR subtypes, M1, M2, M3 and M5 are expressed in the heart. ${ }^{9} \mathrm{M} 2-\mathrm{mAChR}$ is mainly expressed in the atrium; it decreases the level of cAMP and simultaneously activates acetylcholine-sensitive $\mathrm{K}^{+}$channels, exerting a negative chronotropic action. ${ }^{10} \mathrm{M} 3-\mathrm{mAChR}$ is mainly expressed in the ventricle; it shortens the AP duration (APD) of cardiomyocytes via enhancing $\mathrm{K}^{+}$channel currents. ${ }^{11}$ In mice, overexpression of M3-mAChR in the heart reduced the incidence of ventricular arrhythmias, suggesting its role in the prevention of arrhythmias. ${ }^{12} \mathrm{M} 3-\mathrm{mAChR}$ is coupled with $\mathrm{G}_{\mathrm{q}}$ and activates the phospholipase $\mathrm{C}$ (PLC)-inositol triphosphate ( $\left.\mathrm{IP}_{3}\right)$ diacylglycerol (DAG) signaling pathway in the heart, ${ }^{13}$ which consequently activates PKC. ${ }^{14}$ PKC phosphorylates HSF1, leading to increased expression of hsp $70 . .^{15}$

It has been shown that a muscarinic receptor agonist, carbachol, induced PKC-mediated phosphorylation of an E3 ubiquitin ligase, Nedd4-2, thereby inhibited hERG internalization and increased its level on the cell surface. ${ }^{16}$ Maturation of hERG is regulated by the endoplasmic reticulum-associated degradation (ERAD). ${ }^{17}$ It remains unknown whether carbachol could modulate ERAD and the ubiquitin-proteasome pathway.

In the present study, we examined carbachol effects on a novel mutant hERG-FLAG and found that carbachol stabilized the mutant hERG-FLAG through M3-mAChR. This effect was mediated by phosphorylation of HSF1 by PKC and enhanced expressions of hsp70 and hsp90.

\section{Methods}

\section{Genomic Analysis}

This study was approved by the Ethical Committee of the Faculty of Medicine at Tottori University and conformed to the principles outlined in the Declaration of Helsinki. Informed consent for participation in this study was obtained from the subject who was a 15-year-old male. Genomic DNA was extracted from his peripheral blood by using a standard phenolchloroform procedure. A 5.7-kb genomic DNA fragment was amplified by PCR with primers: 5'-CAGGAATTCAGGAGG AGGGTCTAGGAAGTCTTTGGGG-3' and 5'-CCGAAGC TTCTGGGCTAGGAATGGAAGAAGGGGATCC-3'. The digested PCR-amplified fragments with EcoRI and HindIII were sub-cloned into plasmid pTAKN-2 (BioDynamics Laboratory). Resulting plasmids were sequenced with M13 (-21) and M13 reverse primers from both ends.

\section{Expression Plasmid}

cDNA encoding the WThERG was cloned in a mammalian expression vector, pcDNA3.1 (+) (Invitrogen, Carlsbad, CA, USA) and the FLAG tag was ligated at the carboxy terminus of the hERG cDNA. The mutant hERG-FLAG (G572S-D1037_ V1038insGD) cDNA was generated by using a QuikChange site-directed mutagenesis kit (Stratagene).

\section{Cell Culture and Establishment of HEK293 Cell Lines Stably Expressing WT and the Mutant hERG-FLAG}

Cells were cultured in Dulbecco's modified Eagle's medium (D-MEM; Wako), supplemented with $10 \%$ fetal bovine serum (Nichirei Biosciences, Tokyo, Japan) and $0.5 \%$ penicillinstreptomycin $\mathrm{G}$ (Wako) at $37^{\circ} \mathrm{C}$ in a $5 \% \mathrm{CO}_{2}$ incubator. Each plasmid was transfected into HEK293 cells using lipofectamine 2000 (Invitrogen). Stable cell lines were generated by using the antibiotic, G418 (Wako), for selection $(1 \mathrm{mg} / \mathrm{ml})$ and maintenance $(0.4 \mathrm{mg} / \mathrm{ml})$.

\section{Materials}

A M3-mAChR antagonist, diphenylacetoxy- $N$-methylpiperidine methiodide (4-DAMP), and an M2-mAChR antagonist, AF-DX 116 (11[[2-[(diethylamino)methyl]-1-piperidinyl]acetyl]-5,11-dihydro6 $H$-pyrido[2,3-b] [1,4]benzodiazepine6-one), were from Abcam, and a PKC inhibitor, bisindolylmaleimide I hydrochloride (Bis-I), was from Adipogen. These inhibitors were dissolved in dimethyl sulfoxide (DMSO). The final concentration of DMSO in the culture medium was equal to or less than $0.01 \%$ (v/v). Carbamylcholine chloride (Carbachol) from Sigma and other drugs were dissolved in Milli-Q water.

\section{Western Blotting}

Cells were scraped into lysis buffer (PBS containing 1\% NP-40, $0.5 \%$ sodium deoxycholate, $0.1 \%$ SDS, $10 \mu \mathrm{g} / \mathrm{ml}$ aprotinin, $10 \mu \mathrm{g} / \mathrm{ml}$ leupeptin, $10 \mu \mathrm{g} / \mathrm{ml}$ pepstain and $1 \mathrm{mmol} / \mathrm{L}$ phenylmethylsulfonyl fluoride) and lysed by sonication; insoluble materials were removed by centrifugation. Protein concentrations were determined with a protein assay kit (Bio-Rad). Ten $\mu \mathrm{g}$ of proteins were separated on SDS-PAGE and electrotransferred to a PVDF membrane (Immobilon-P; Millipore). Membranes were probed with antibodies against FLAG (Agilent Technologies), $\alpha$-tubulin (Abcam), hsp70 (Enzo Life Sciences), hsp90 (Santa Cruz), Ser326-phosphorylated HSF1 (Abcam), HSF1 (Merck Millipore), M3-mAChR (Santa Cruz), M2-mAChR (Santa Cruz) and were developed using an ECL system (Amersham). Band intensities were quantified using Image $\mathbf{J}$ software.

\section{Chase Assay}

HEK293 cells stably expressing WT or the mutant hERGFLAG were seeded in 6-well plates. Brefeldin A (Sigma) was administrated to cells for $12 \mathrm{~h}$ before carbachol treatment. After the addition of cycloheximide $(60 \mu \mathrm{g} / \mathrm{ml})$, protein extracts were prepared at the indicated times and subjected to antiFLAG Western blotting.

\section{Immunofluorescence}

HEK293 cells were seeded on gelatin-coated coverslips and transfected with WT or the mutant hERG-FLAG plasmid, together with pDsRed2-ER (Clontech, Mountain View, CA, USA), pDsRed-Monomer-Golgi (Clontech) or pPM-mKeimaRed (BML, Tokyo, Japan). All the staining procedures were conducted at room temperature. Twenty-four hours after transfection, cells were fixed with $4 \%$ paraformaldehyde for $10 \mathrm{~min}$, 


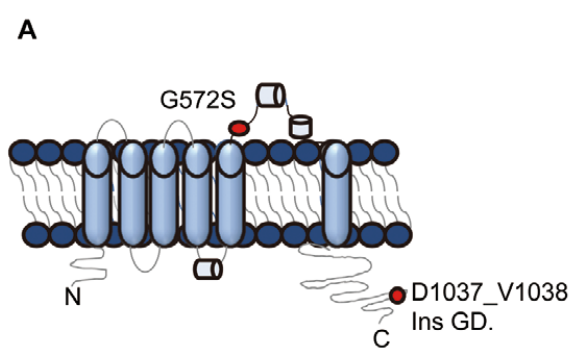

C

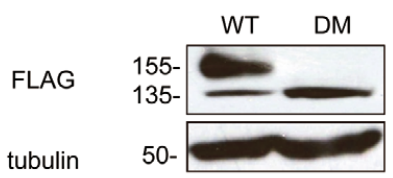

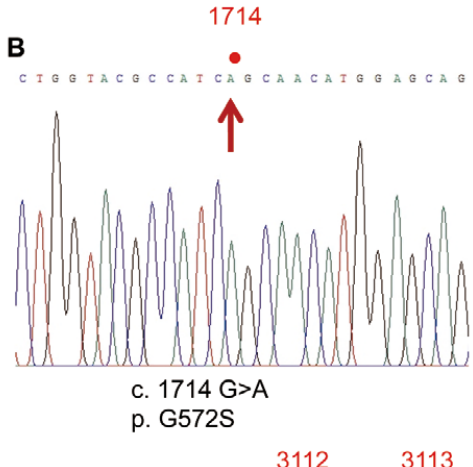

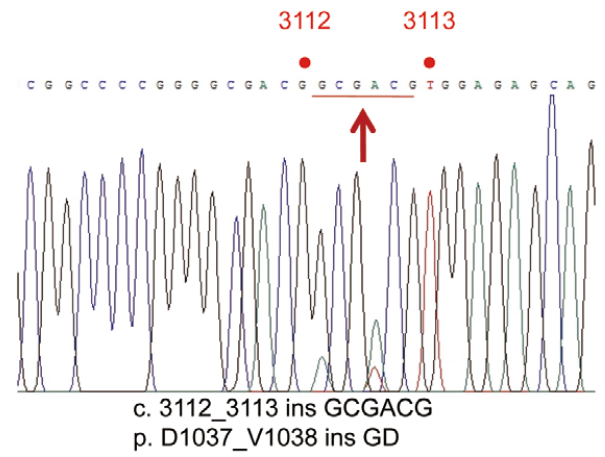

Figure 1. Characterization of a novel mutant hERG-FLAG. (A) Locations of G572S and D1037_V1038insGD in the human ethera-go-go-related gene (hERG). (B) Sequence analysis of exon 7 and exon 13. A point mutation A to G 1714 in $h E R G$ causes substitution G572S. An insertion of GCGACG between the 3112 and 3113 in $h E R G$ yields the insertion D1037_V1038insGD. (C) Western blots of WThERG-FLAG (wild-type; WT) and the mutant hERG-FLAG with the double mutation G572S-D1037_V1038insGD, denoted as DM. Lysates from HEK293 stably expressing WT or DM were subjected to immunoblotting (IB) with indicated antibodies.

permeabilized with $0.5 \%$ Triton X-100 for $10 \mathrm{~min}$, and blocked with 3\% albumin in PBS for 30 min. Cells were incubated for $1 \mathrm{~h}$ with the anti-FLAG antibody (Agilent Technologies). Bound antibodies were visualized with AlexaFluor 488-conjugated mouse secondary antibody, and images were obtained by using a Bio-Rad MRC 1024 confocal microscope. To quantify hERG-FLAG signals, images were cropped with regard to the distribution of each marker protein (DsRed2-ER, DsRedMonomer-Golgi or PM-mKeima-Red) using Photoshop CS3 software. Signal intensities in cropped areas were quantified by using Image $\mathrm{J}$ software.

\section{Reporter Gene Assay}

A plasmid encoding a luciferase gene driven by the hsp70 promoter (phsp70 firefly luciferase) was provided by A. Nakai. HEK293 cells expressing WT and the mutant hERG-FLAG were prepared in 96 wells at $70-80 \%$ confluencies. CMVRenilla luciferase plasmid (pCMV-Renilla luciferase) (Promega, WI, USA) and phsp70 firefly luciferase were co-transfected into the cells. After $24 \mathrm{~h}$ of transfection, cells were treated with or without carbachol. The luciferase activity in the cell lysates was determined by using Dual-Glo ${ }^{\circledR}$ (Promega), according to the manufacturer's instructions. The results were expressed as the hsp70 luciferase activity normalized to Renilla luciferase activity.

\section{Electrophysiological Recordings}

The hERG channel currents ( $I_{\mathrm{hERG}}$ ) corresponding to $I_{\mathrm{Kr}}$ were measured by whole-cell patch-clamp techniques with an Axopatch-200B amplifier (Molecular Devices). The extracel- lular solution contained (mmol/L): $\mathrm{NaCl} 140, \mathrm{KCl} 4, \mathrm{CaCl}_{2}$ $1.8, \mathrm{MgCl}_{2} 0.53, \mathrm{NaH}_{2} \mathrm{PO}_{4} 0.33$, glucose 5.5, and HEPES 5 $(\mathrm{pH}$ 7.4). The internal pipette solution contained (mmol/L): K-aspartate 100, $\mathrm{KCl} 20, \mathrm{CaCl}_{2}$ 1, Mg-ATP 5, EGTA 5, HEPES 5 , and creatine phosphate dipotassium salt $5(\mathrm{pH} 7.2)$. Patch pipettes had a resistance of 2-4 M 2 . After rupture of the cell membrane, whole-cell membrane currents were recorded at $37^{\circ} \mathrm{C}$ under the voltage-clamp mode. Series resistance $\left(\mathrm{R}_{\mathrm{s}}\right)$ was determined by fitting a single exponential function to the capacitive current decay to estimate its time constant $(\tau)$ and the membrane capacitance $\left(\mathrm{C}_{\mathrm{m}}\right)$; Rs calculated with the equation $\mathrm{R}_{\mathrm{s}}=\tau / \mathrm{C}_{\mathrm{m}}$ during the capacitive current cancellation ranged $2.2 \pm 0.4 \mathrm{M} \Omega$ with $\tau=98 \pm 8 \mu \mathrm{s}$ and $\mathrm{C}_{\mathrm{m}}=46 \pm 24 \mathrm{pF}(\mathrm{n}=5)$. After the $\mathrm{R}_{\mathrm{s}}$ compensation of $50-60 \%$, the voltage errors arising from $R_{s}$ were estimated to be less than $5 \mathrm{mV}$. The membrane potential was not corrected for the liquid junction potential, which was estimated to be $<10 \mathrm{mV}$. Holding potential was set at $-80 \mathrm{mV}$ and $\mathrm{ILRG}_{\mathrm{hER}}$ was evoked by 2 -s depolarizing test pulses ranging from -50 to $+50 \mathrm{mV}$ (with a $10-\mathrm{mV}$ increment) followed by repolarization to $-60 \mathrm{mV}$ for $6 \mathrm{~s} .{ }^{18} \mathrm{~A}$ blocker selective for hERG channels, E4031 (Wako), was added at $10 \mu \mathrm{mol} / \mathrm{L}$; E4031-sensitive currents were measured by digital subtraction of the current traces with and without E4031. The peak current amplitudes were determined during test pulses and plotted as functions of depolarizing pulses.

\section{Computer Simulations of APs of Human Ventricular Myocytes (HVMs)}

We simulated prolongation of APDs and generation of early afterdepolarization (EAD) in LQT2 HVMs with the mutant 

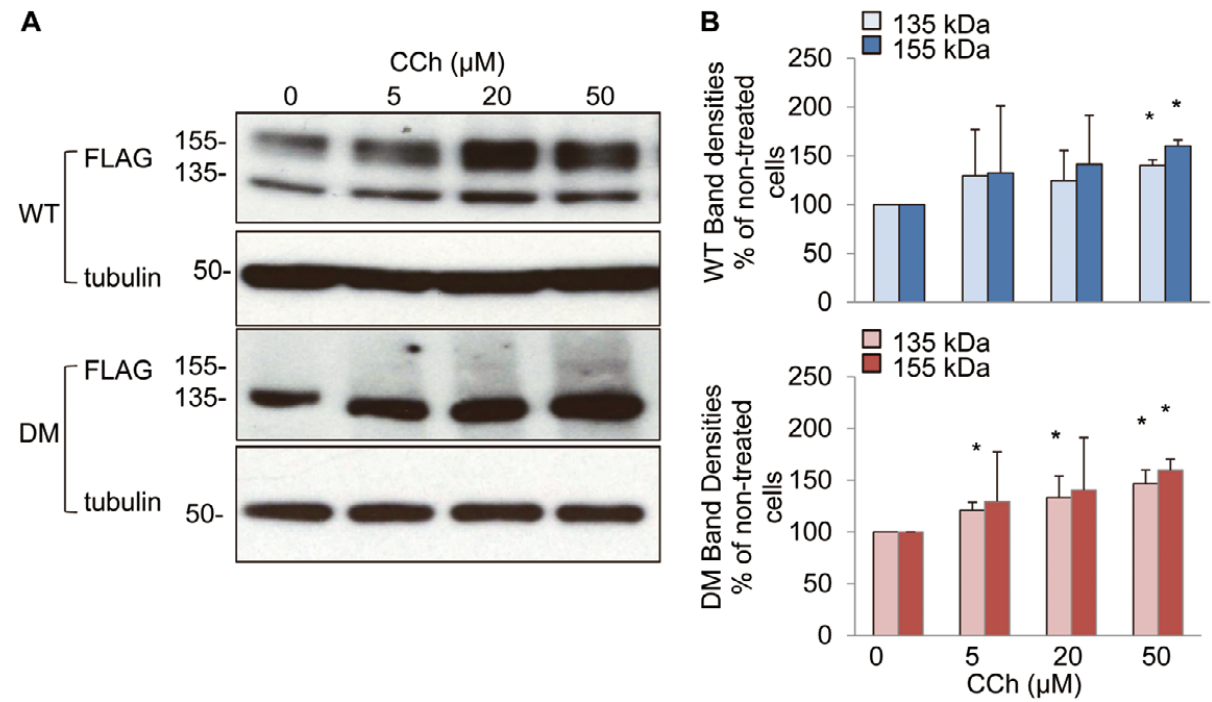

Figure 2. Effects of carbachol (CCh) on the expression of WThERG-FLAG and the mutant hERG-FLAG. (A) Representative blots of the WThERG-FLAG and mutant hERG-FLAG expressed in HEK293 cells in the absence and presence of 5-50 $\mu \mathrm{mol} / \mathrm{L}$ CCh. HEK293 stably expressing the wild-type (WT) or double mutation (DM) were treated with CCh at the indicated concentrations for $2 \mathrm{~h}$. Cell lysates were subjected to immunoblotting (IB) with indicated antibodies. (B) Averaged densities of hERG-FLAG proteins with or without the CCh treatment. The band densities of hERG-FLAG proteins were normalized to that of tubulin, and then their densities in the presence of 5-50 $\mu \mathrm{mol} / \mathrm{L}$ carbachol was normalized to that in the absence of $\mathrm{CCh}(\mathrm{CCh}=0)$. ${ }^{*} \mathrm{P}<0.05 \mathrm{vs}$. CCh=0 $(n=5$ each).

hERG using mathematical models developed by Kurata et $\mathrm{al}^{19}$ and O'Hara et al. ${ }^{20}$ A mid-myocardial (M) cell version of the Kurata et al model was developed on the basis of the transmural heterogeneity in densities of sarcolemmal ion channels, transporters and $\mathrm{SR} \mathrm{Ca}^{2+}$ uptake/release rates, as summarized by O'Hara et al. ${ }^{20} \mathrm{We}$ used the $\mathrm{M}$ cell versions of the two HVM models because they have larger ICaL, as well as smaller $I_{\mathrm{Kr}}$ and $I_{\mathrm{Ks}}$, thus being much more vulnerable to EAD formation than the endocardial and epicardial versions. ${ }^{20}$ Whether the carbachol-induced modification of the mutant hERG current inhibits EAD formation in the LQT2 HVMs was tested, as shown in the supplementary material.

\section{Statistical Analysis}

All the data were presented as mean \pm SEM, and analyzed by using a Student's $t$-test or repeated measures analysis of variance (2-way ANOVA). The differences between groups were considered statistically significant at $\mathrm{P}<0.05$.

\section{Results}

Characterization of the Mutant hERG-FLAG Channel Protein Two mutations of hERG were found in the genome of a LQT2 patient with a QTC of $520 \mathrm{~ms}$ (Figure 1A). One causes the amino acid substitution G572S and the other causes the amino acid insertion D1037_V1038insGD (Figure 1B). Pedigree and ECGs of the patient and his family are shown in the Figure S1. Next, we examined whether the 2 mutations beside in the same. Genomic DNA fragments that contained both mutation sites were amplified by PCR. Ten colonies were randomly chosen and the sub-cloned DNA fragments were sequenced. Sequencing of 7 colonies revealed both mutations resided in a genomic fragment while 3 colonies represented the WT hERG sequence, indicating that the patient had a double mutant in the single allele. When expressed in HEK293 cells, WThERG-FLAG gave a $155-\mathrm{kDa}$ mature and a $135-\mathrm{kDa}$ immature forms, whereas the mutant hERG-FLAG exclusively remained in its immature form (Figure 1C).

It has been reported that the G572S single mutant exerted a dominant negative effect. ${ }^{21}$ Therefore, we examined whether the double mutant has the same effect by co-expression as reported previously. Co-expression of G572S resulted in a drastic decrease in the E-4031-sensitive currents. This effect was observed in the double mutant and was barely observed with the insertion. Thus, the double mutant also has the dominantnegative effect, albeit much less than G572S (Figure S2).

Carbachol Facilitates Maturation of the Mutant hERG-FLAG We examined carbachol effects on hERG maturation in HEK293 cells stably expressing hERG-FLAG. Carbachol caused dosedependent increases of both mature and immature forms of the mutant hERG-FLAG (DM), as well as WT (Figures 2A,B). Time-course analysis showed that a $2 \mathrm{~h}$ treatment with carba$\mathrm{chol}$ at $50 \mu \mathrm{mol} / \mathrm{L}$ was enough to induce the maximum effect (Figures S3A,B). Carbachol also increased the mature form of another mutant hERG G601S-FLAG (Figure S4).

Immunofluorescence showed that the signals of both the WT and the mutant hERG-FLAG co-localized with those of the ER $(\# 3,12)$ (Figure 3A). The signals of the mutant hERGFLAG were slightly decreased in the Golgi apparatus (\#15) and were absent on the PM (\#18). Treatment with carbachol significantly increased the signals of the mutant hERG-FLAG in the Golgi apparatus (\#24) as well as on the PM (\#27). The changes in immunoreactivities were confirmed by quantification of the signal activities (Figure 3B).

Figure $4 \mathrm{~A}$ shows the original traces of E4031-sensitive 

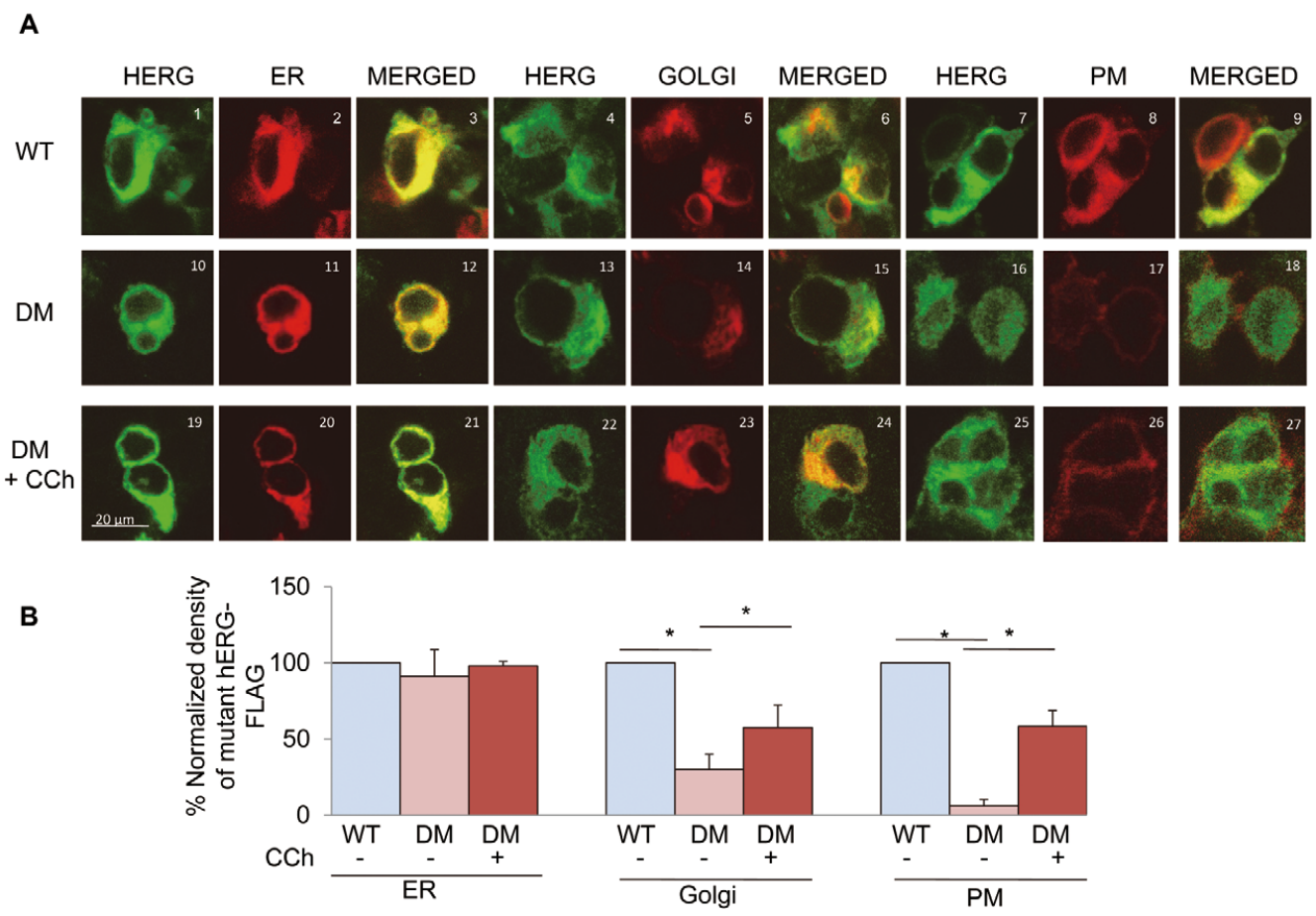

Figure 3. Carbachol (CCh) treatment promotes the protein expression of the mutant hERG-FLAG in the Golgi apparatus and on the cell surface. (A) Immunofluorescence of wild-type (WT) and the mutant hERG-FLAG proteins in the presence (DM+CCh) and absence (double mutation; DM) of 50 mol/L carbachol in HEK293 cells co-expressing DsRed2-ER (ER), DsRed-Monomer-Golgi (GOLGI) or PM-mKeima-Red (PM). All cells were stained with anti-FLAG and Alexa Fluor 488-conjugated mouse secondary antibody (green). Shown are representative images for hERG-FLAG and individual marker proteins, as well as merged images, obtained by using a confocal microscope. (B) A histogram summarizing the ratios of Alexa 488/DsRed-ER, DsRed-Monomer-Golgi and PM-mKeima-Red fluorescence. The signals of WT and the DM protein merged with those of the marker proteins were normalized to WT and the DM signals, respectively, and the densities of the DM in each compartment were expressed as the values relative to those of the WT. ${ }^{*} \mathrm{P}<0.05$ ( $\mathrm{n}=4$ each).

A

WT

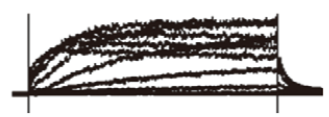

DM

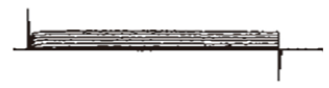

DM

$+\mathrm{CCh}$

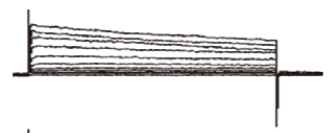

HEK

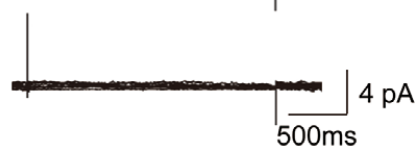

B

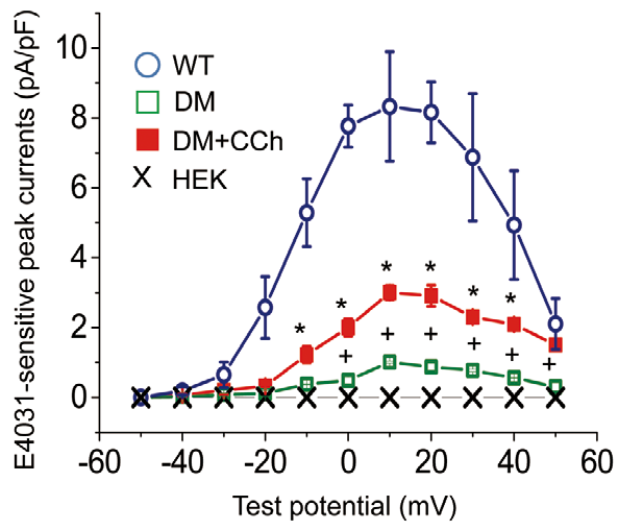

Figure 4. Carbachol (CCh) effects on hERG currents in HEK293 cells stably expressing wild-type (WT) or the mutant hERG-FLAG. (A) Representative traces of E-4031-sensitive currents recorded in the WT, mutant hERG-FLAG in the absence (double mutation; $\mathrm{DM})$ and presence of $50 \mu \mathrm{mol} / \mathrm{L}(2 \mathrm{~h})$ carbachol (DM+CCh) and those of HEK293 cells transfected with mock plasmid (HEK). During the experiment, $10 \mu \mathrm{mol} / \mathrm{L}$ E-4031 was added. E-4031 sensitive currents were obtained by digital subtraction. (B) Currentvoltage relationships for E-4031-sensitive peak currents determined during the 2-s depolarizing pulses. Differences were tested for statistical significance by using a 2-way ANOVA. ${ }^{*} \mathrm{P}<0.05$ vs. WT, ${ }^{+} \mathrm{P}<0.05$ vs. DM ( $\mathrm{n}=10-12$ each). 


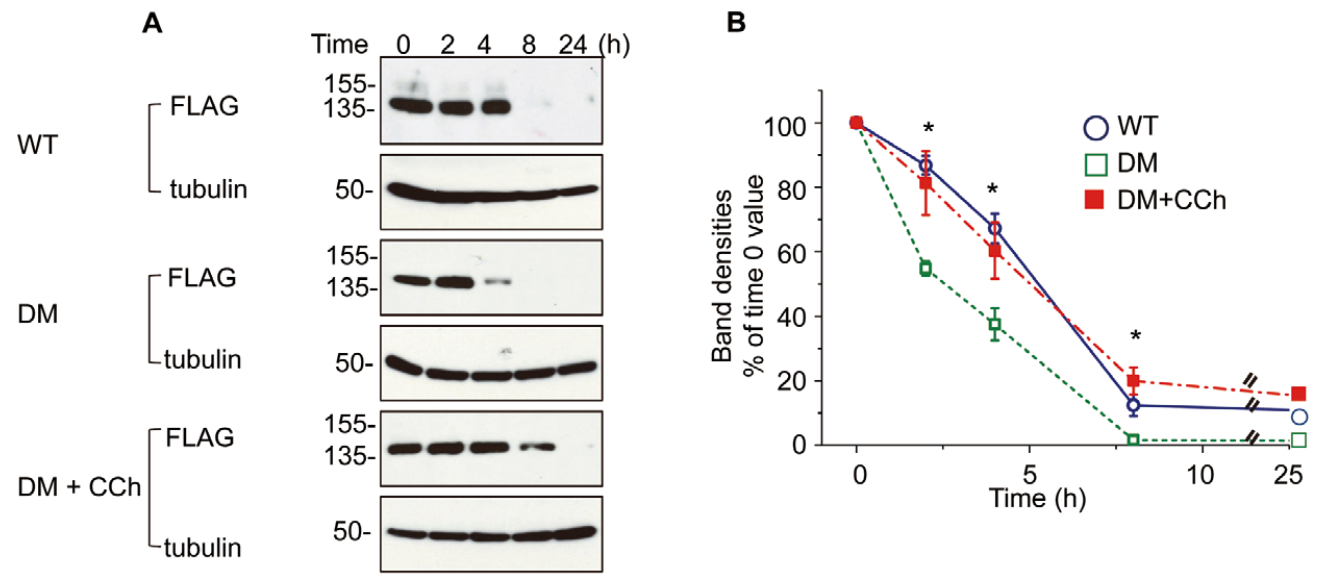

Figure 5. Carbachol promotes hERG-FLAG protein maturation in the presence of brefeldin A. Degradation of the wild-type (WT) and the mutant hERG-FLAG (double mutation; DM) proteins in comparison with $50 \mu \mathrm{mol} / \mathrm{L}$ CCh treatment in the presence of brefeldin A. HEK293 cells stably expressing the WT and DM were treated with $10 \mu \mathrm{mol} / \mathrm{L}$ brefeldin A for $12 \mathrm{~h}$ before carbachol treatment. Cycloheximide was added, and cell lysates were taken at indicated times; cell lysates were subjected to immunoblotting (IB) with indicated antibodies (A,B). ${ }^{*}<0.05$ vs. the DM only ( $n=5$ each).

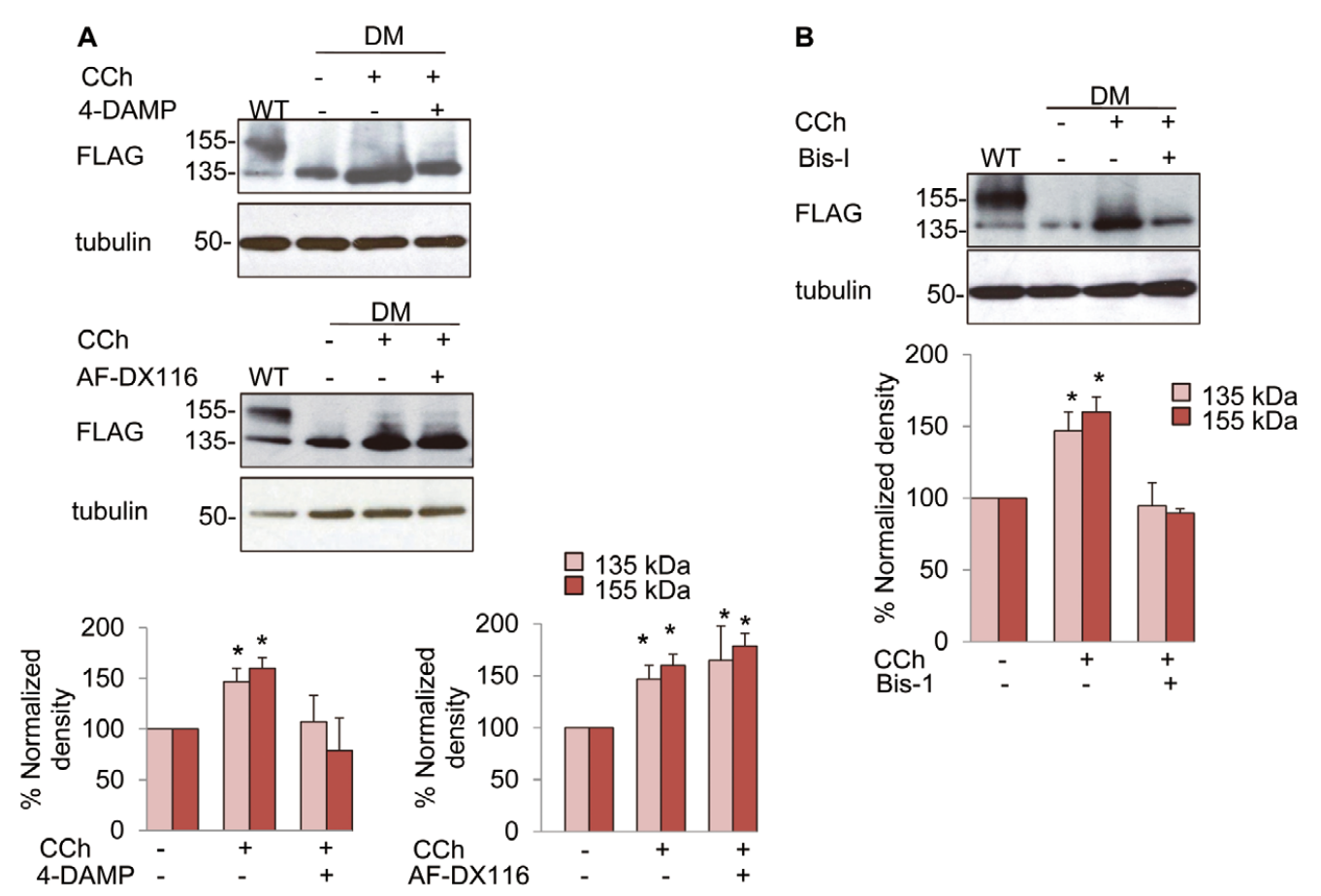

Figure 6. Carbachol (CCh) promotes the mutant hERG-FLAG maturation through activation of the M3 muscarinic receptor and PKC. HEK293 cells stably expressing the mutant hERG-FLAG (double mutation; DM) were treated with $50 \mu \mathrm{mol} / \mathrm{L}$ CCh for $2 \mathrm{~h}$ in the absence or presence of $1 \mu \mathrm{mol} / \mathrm{L}$ 4-DAMP, $1 \mu \mathrm{mol} / \mathrm{L}$ AF-DX116 (A) or $3 \mu \mathrm{mol} / \mathrm{L}$ Bis-I (B). Cell lysates were subjected to immunoblotting (IB) with indicated antibodies. Bar graphs show the averaged densities of hERG-FLAG proteins, which were normalized to that of tubulin and then that in the absence of carbachol (CCh) and the antagonists or the inhibitor. ${ }^{*}<<0.05$ vs. $D M$ only ( $n=5$ each). 
A
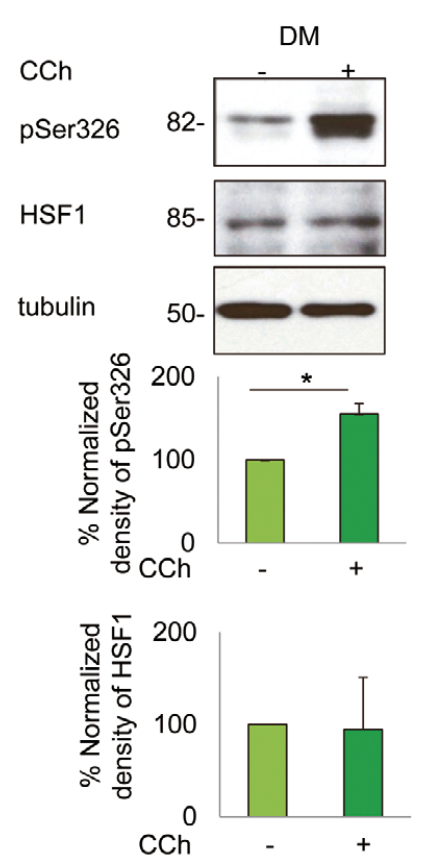

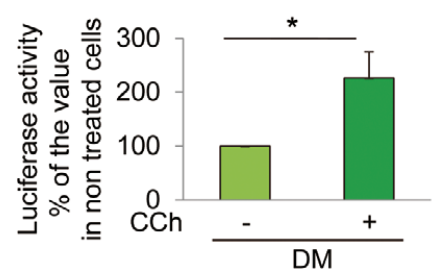

C

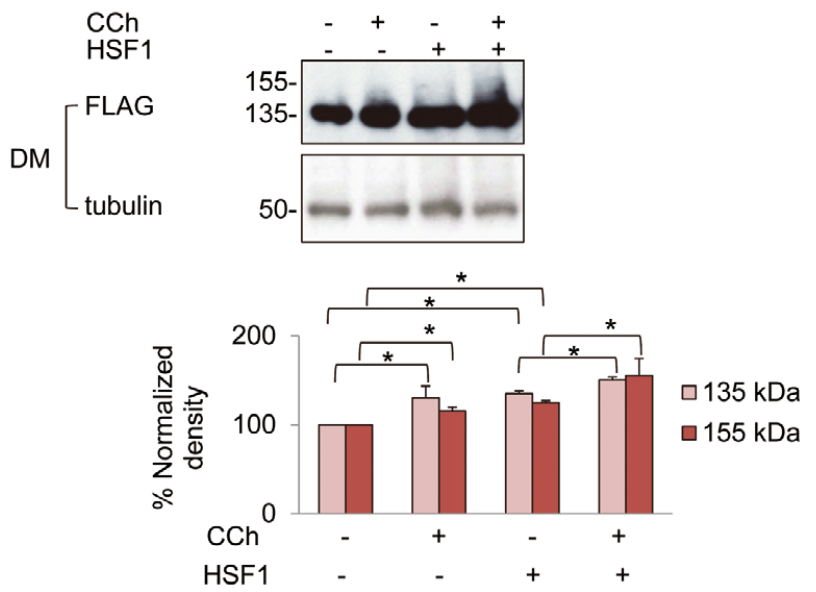

Figure 7. Carbachol (CCh) facilitates phosphorylation of heat shock factor 1 (HSF1) associated with the maturation of the mutant hERG-FLAG. (A) Effects of $50 \mu \mathrm{mol} / \mathrm{L}$ CCh on the phosphorylated HSF1 at the 326 serine residue (pSer326). HEK293 cells stably expressing the mutant hERG-FLAG (double mutation; DM) were treated with CCh for $2 \mathrm{~h}$. Cell lysates were subjected to immunoblotting (IB) with indicated antibodies. The bar graphs show the averaged values normalized to those of tubulin and to those in the absence of CCh. ${ }^{*} \mathrm{P}<0.05$ ( $n=5$ each). (B) Luciferase assay result for hsp70 promoter with or without $50 \mu$ mol/L CCh treatment. CMV-Renilla luciferase plasmid (pCMV-Renilla luciferase) and phsp70 firefly luciferase were co-transfected with the DM into the HEK293 cells; $24 \mathrm{~h}$ after transfection, cells were treated with (+) or without CCh (-). The luciferase activity was measured from the cell lysates. A bar graph shows the averaged values normalized to those in the absence of CCh. ${ }^{*}<0.05$ ( $n=5$ each). (C) Effects of $50 \mu \mathrm{mol} / \mathrm{L} \mathrm{CCh}$ and HSF1 on the DM. HEK293 cells were transfected with the DM and HSF1 plasmids (+) or pcDNA3 (-). Twentyfour hours after transfection, cells were treated with $50 \mu \mathrm{mol} / \mathrm{L}$ CCh. Cells lysates were subjected to immunoblotting (IB) with indicated antibodies. A bar graph shows the averaged amounts of $h E R G-F L A G$ proteins, which were normalized to those without CCh treatment and HSF1. ${ }^{*}<0.05$ ( $n=3$ each).

currents recorded in HEK293 cells stably expressing the WT or mutant hERG-FLAG. Depolarizing pulses activated timedependent outward currents that corresponded to $I_{\mathrm{Kr}}$ in cells expressing WThERG-FLAG. Cells expressing the mutant hERG-FLAG showed substantially reduced E4031-sensitive currents. At $+10 \mathrm{mV}$, the peak currents were decreased by $\sim 80 \%$ compared with the currents in cells expressing the WT. Treatment with carbachol increased the currents (Figure 4B). These results suggested that carbachol enhanced maturation of the mutant hERG-FLAG proteins, which are then transported to the cell surface.

Carbachol appeared to facilitate maturation of the mutant hERG-FLAG. To see whether carbachol could affect ERAD of the mutant hERG-FLAG, we examined the effects of carbachol in the presence of brefeldin A, which blocks protein transport from the ER to the Golgi apparatus, and cycloheximide, which blocks the protein synthesis. Degradation of the mutant hERG-FLAG protein was faster than that of WThERG-FLAG, and carbachol suppressed its degradation (Figures 5A,B). Thus, carbachol could stabilize the mutant hERG-FLAG by suppressing its ERAD.

\section{Carbachol Facilitates Maturation of the Mutant hERG-FLAG Through Activation of M3-mAChR}

To identify the mAChR subtype responsible for the carbachol effects, we used a M3-mAChR antagonist, 4-DAMP, and a M2-mAChR antagonist, AF-DX 116, because HEK293 cells expressed both M2-mAChR and M3-mAChR (Figure S5A). 4-DAMP, but not AF-DX 116, abolished the effect of carbachol on the protein levels of the mutant hERG-FLAG (Figure 6A) as well as WThERG-FLAG (Figures S5B,C), indicating the involvement of M3- but not M2-mAChR.

In order to examine whether the PKC pathway is involved in the action of carbachol, we studied the effects of a PKC inhibitor, Bis-I, on the carbachol-induced maturation of the mutant hERG-FLAG. Bis-I at $3 \mu \mathrm{mol} / \mathrm{L}$ abolished the carbachol effects on the mutant hERG-FLAG (Figure 6B) as well as on WThERG-FLAG (Figure S6A).

\section{Carbachol Facilitates Maturation of the Mutant hERG-FLAG Through Phosphorylation of HSF1}

Both Hsp70 and hsp90 facilitate maturation of the WThERGFLAG and mutant hERG-FLAG. ${ }^{7,8}$ Expressions of both hsps are regulated by HSF1, which is one of the downstream effec- 

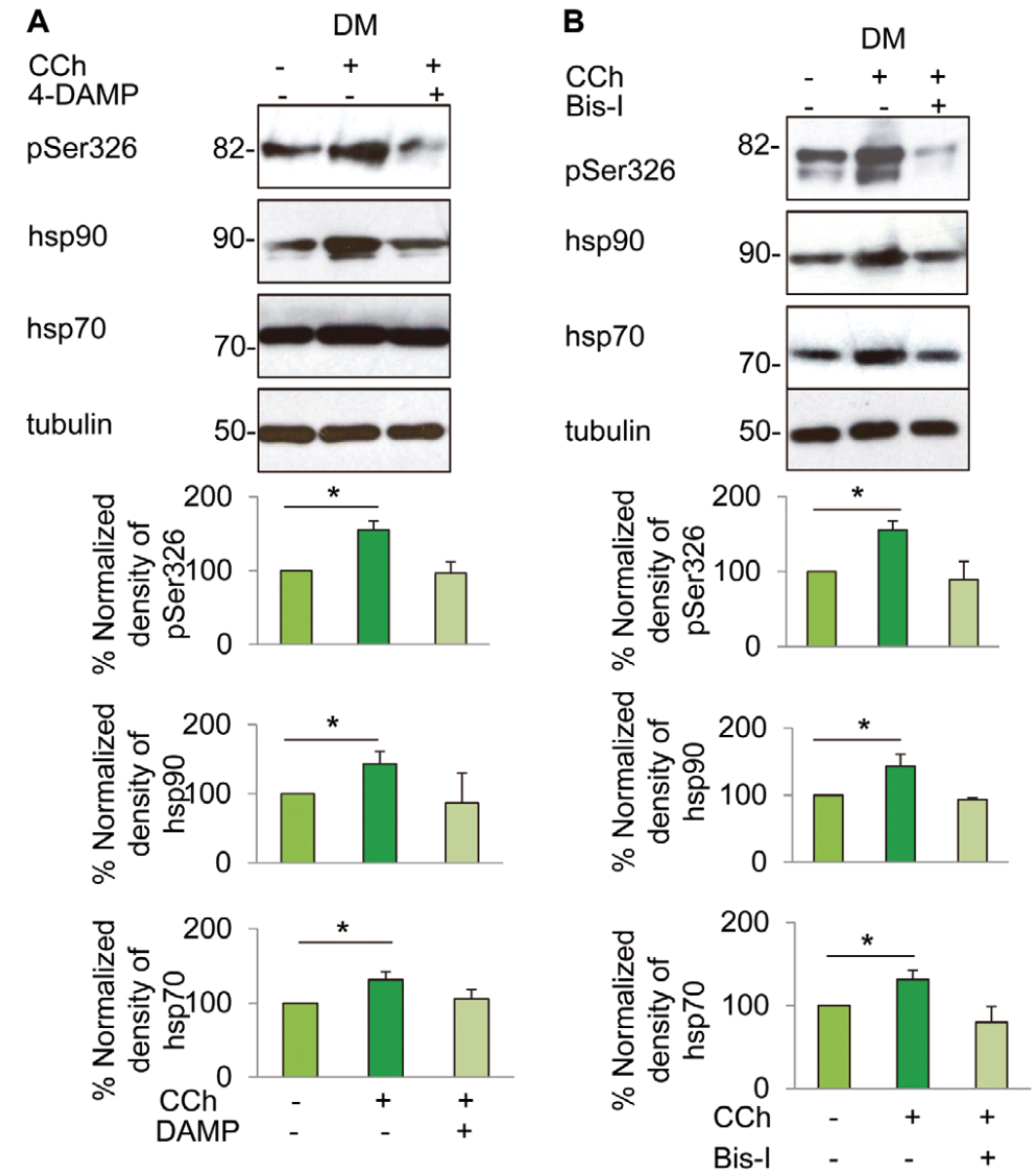

Figure 8. Carbachol (CCh) increases heat shock protein expression through the $\mathrm{M} 3$ receptor-PKC dependent pathway. Effects of $50 \mu \mathrm{mol} / \mathrm{L}$ CCh on phosphorylated HSF1 and expressions of hsp90 and hsp70 in the absence or presence of $1 \mu \mathrm{mol} / \mathrm{L}$ 4-DAMP (A) or $3 \mu \mathrm{mol} / \mathrm{L}$ Bis-I (B) in HEK293 cells stably expressing the mutant hERG-FLAG (double mutation; DM). The cells were treated with $\mathrm{CCh}$ for $2 \mathrm{~h}$. Bar graphs show the averaged band densities of pSer326, hsp70 and hsp90 normalized to those of tubulin and those in the absence of CCh. ${ }^{*} \mathrm{P}<0.05$ ( $n=5$ each).

tors of PKC. ${ }^{5,15}$ To examine whether carbachol could induce phosphorylation of HSF1, we used an antibody that recognizes HSF1 with a phosphorylated serine residue at aa 326 (pSer326). Treatment with carbachol increased the level of pSer326 in cells expressing the mutant hERG-FLAG without changes in the total protein level of HSF1 (Figure 7A).

To confirm the role of the HSF1-hsp pathway, we examined whether carbachol could increase the transcription of hsp 70 by using a reporter gene assay. Treatment with carbachol increased transcription of the hsp70 reporter gene (Figure 7B) and enhanced the protein levels of hsp70 and hsp90 (Figures 8A,B). Moreover, carbachol could further facilitate maturation of the mutant hERG-FLAG when cells were co-transfected with HSF1 as well as the WThERG-FLAG (Figure 7C; Figure S6B).

In accordance with these findings, inhibition of the M3-mAChR-PKC pathway by 4-DAMP $(1 \mu \mathrm{mol} / \mathrm{L})$ or Bis-I $(3 \mu \mathrm{mol} / \mathrm{L})$ abolished carbachol-induced increases of hsp70 and hsp90, as well as pSer326 (Figures 8A,B).

\section{Discussion}

We found novel double mutations of the hERG gene in a Japanese male LQT2 patient; one was an amino acid substitution, G572S, in the pore domain and the other was an inframe insertion of D1037_V1038insGD in the carboxyl terminus. The mutant hERG-FLAG was expressed solely as an immature form at $135 \mathrm{kDa}$. It was localized predominantly in the ER and was not expressed on the cell surface. Carbachol induced the mature form $(155 \mathrm{kDa})$ of the mutant hERG-FLAG. We demonstrated that carbachol activated M3-mAchR to phosphoryilate HSF1 via activation of the PKC, resulting in activation of hsp family proteins and maturation of the mutant hERG-FLAG. Carbachol has been reported to increase the mature form of WThERG-FLAG via inhibition of its internalization, and the current study is the first report to demonstrate that the same drug could facilitate maturation of the mutant hERG-FLAG at the ER.

Muscarinic receptors mediate parasympathetic nerve actions on the heart. M1-, M2-, M3- and M5-mAChR have been reported to be expressed in the human heart, and each of them has different distribution and function. ${ }^{9} \mathrm{M} 3-\mathrm{mAChR}$ is predominantly expressed in the ventricle and exerts negative inotropic and chronotropic effects. It also regulates cardiac AP repolarization and cell-to-cell communication. ${ }^{22}$ Two subtypes of muscarinic receptors, M2- and M3-mAChR, have been reported to be expressed in HEK293 cells. ${ }^{23}$ The M3-mAChR antagonist, 4-DAMP, but not the M2-mAChR antagonist, AF-DX116, abolished carbachol-induced maturation of the mutant hERG-FLAG, indicating the involvement of M3-mAChR. The role of PKC was evidenced by the Bis-I effect on carbachol-induced maturation of the mutant hERG-FLAG. Previous reports show that M3-mAChR could activate $P K C$ through $G_{q}$ proteins. $^{24}$

It has been reported that carbachol induces phosphorylation 
of Nedd4-2 via the PKC pathway through M3-mAChR, inhibits monoubiquitination of WThERG-FLAG and suppresses its internalization from the PM. This results in inhibition of its degradation in the endosome and an increase of channel function. ${ }^{16,25} \mathrm{We}$ showed that carbachol facilitated maturation of the trafficking-deficient mutant hERG-FLAG via the PKC pathway through activation of M3-mAChR, and that this effect was mediated by phosphorylation of HSF1. HSF1 is activated by 2 steps. On the first step, HSF1 binds to HSEs and forms an oligomer; and on the second step, it is phosphorylated to exert a high transcriptional activity. ${ }^{5}$ The serine residue at position 326 in HSF1 has been identified as a dominant target for phosphorylation regulators. ${ }^{26}$ As shown in Figure 7, carbachol increased phosphorylation of Ser326 in HSF1 and activated the promoter activity of hsp70. This subsequently increased protein levels of hsp70 and hsp90. Overexpression of HSF1 enhanced the maturation of the hERG protein, providing further evidence for the role of HSF1.

Our novel mutant hERG-FLAG yielded very small currents and had a dominant negative effect. The currents mediated by the mutant hERG-FLAG appear to be much faster than that of the WThERG-FLAG, suggesting that the mutant channel has distinct gating properties. Therefore, carbachol may not be beneficial in shortening APDs or inhibiting EAD formation, especially if the mutant proteins harbor severe gating abnormalities. Thus, we tested whether the carbachol-induced increase in the mutant channels could lead to APD shortening by computer simulation using the Kurata et al and O'Hara et al models for HVMs. ${ }^{19,20}$ Both models predicted that the carbachol-induced increase in the mutant hERG-FLAG could yield APD shortening in HVMs expressing the mutant hERG (Figure S7). Thus, the enhancement of the mutant hERG-FLAG current by carbachol could be beneficial for certain LQT2 patients.

There are some limitations in our study, which are as follows: (1) only the proband exhibited the prominent prolongation of QTc, suggesting that the G572S-D1037_V1038insGD mutation in 1 allele may be a de novo mutation. Unfortunately, other family members' genomic information on the hERG gene was lacking because of their refusal to our proposal for genetic analysis; and (2) we used the HEK293 cells stably expressing hERG channels to examine the effects of carbachol on the maturation of the mutant hERG-FLAG, because the HEK293 cells are well known to be capable of characterizing cardiotoxic agents that are involved in prolongation of QT intervals and display biophysical and pharmacological properties similar to those in native cardiomyocytes. However, the heterologous expression system is not identical to native cardiomyocytes in terms of trafficking and degradation of the hERG protein. Cell-specific post-transcriptional and post-translational modifications are very important for channel functions, as suggested recently. ${ }^{27}$ Carbachol facilitated the maturation of endogenous ERG in HL-1 cells as well as the mutant hERGFLAG in transfected HL-1 cells (Figure S8). Nevertheless, further experiments using iPS cell-derived cardiomyocytes originated from LQT2 patients will be necessary.

\section{Acknowledgments / Conflicts of Interest}

None.

\section{References}

1. Sanguinetti MC, Jiang C, Curran ME, Keating MT. A mechanistic link between an inherited and an acquired cardiac arrhythmia: $H E R G$ encodes the IKr potassium channel. Cell 1995; 81: 299-307.

2. Wilde A, Tan HL. Inherited arrhythmia syndromes. Circ J 2007; 71:
$12-19$

3. Nishizaki M, Hiraoka M. Gene mutations associated with atrioventricular block complicated by long QT syndrome. Circ J 2010; 74: 2546-2547.

4. Anderson CL, Delisle BP, Anson BD, Kilby JA, Will ML, Tester DJ, et al. Most LQT2 mutations reduce Kv11.1 (hERG) current by a class 2 (trafficking-deficient) mechanism. Circulation 2006; 113: $365-373$.

5. Sorger PK. Heat shock factor and the heat shock response. Cell 1991; 65: $363-366$.

6. Ficker E, Dennis AT, Wang L, Brown AM. Role of the cytosolic chaperones Hsp70 and Hsp90 in maturation of the cardiac potassium channel hERG. Circ Res 2003; 92: e87-e100, doi:10.1161/01. RES.0000079028.31393.15.

7. Li P, Ninomiya H, Kurata Y, Kato M, Miake J, Yamamoto Y, et al. Reciprocal control of hERG stability by Hsp70 and Hsc70 with implication for restoration of LQT2 mutant stability. Circ Res 2011; 108: $458-468$.

8. Iwai C, Li P, Kurata Y, Hoshikawa Y, Morikawa K, Maharani N, et al. Hsp90 prevents interaction between CHIP and HERG proteins to facilitate maturation of wild-type and mutant HERG proteins. Cardiovasc Res 2013; 100: 520-528.

9. Wang H, Han H, Zhang L, Shi H, Schram G, Nattel S, et al. Expression of multiple subtypes of muscarinic receptors and cellular distribution in the human heart. Mol Pharmacol 2001; 59: 1029-1036.

10. Kitazawa T, Teraoka H, Harada N, Ochi K, Nakamura T, Asakawa $\mathrm{K}$, et al. Regulation of heart contractility by M2 and M3 muscarinic receptors: Functional studies using muscarinic receptor knockout mouse. In: Myslivecek J, Jakubik J, editors. Muscarinic receptor: From structure to animal models. New York: Springer, 2016, 235-259.

11. Shi H, Wang H, Wang Z. Identification and characterization of multiple subtypes of muscarinic acetylcholine receptors and their physiological functions in canine hearts. Mol Pharmacol 1999; 55: 497-507.

12. Liu Y, Sun L, Pan Z, Bai Y, Wang N, Zhao J, et al. Overexpression of M3 muscarinic receptor is a novel strategy for preventing sudden cardiac death in transgenic mice. Mol Med 2011; 17: 1179-1187.

13. Mathes PW, Leineweber K, Wangemann T, Silber RE, Brodde OE. Existence of functional M3-muscarinic receptors in the human heart. Naunyn Scmiedebergs Arch Pharmacol 2003; 368: 316-319.

14. Shi H, Wang H, Yang B, Xu D, Wang Z. The M3 receptor-mediated $\mathrm{K}^{+}$current (I км3) a Gq protein-coupled $\mathrm{K}^{+}$channel. J Biol Chem 2004; 279: 21774-21778

15. Yamanaka K, Takahashi N, Matsumoto H, Ohnishi T, Kaneda K, Yoshimatsu $\mathrm{H}$, et al. Role of protein kinase $\mathrm{C}$ in geranylgeranylacetone-induced expression of heat-shock protein 72 and cardioprotection in the rat heart. J Mol Cell Cardiol 2003; 35: 785-794.

16. Wang T, Hogan-Cann A, Kang Y, Cui Z, Guo J, Yang T, et al. Muscarinic receptor activation increases hERG channel expression through phosphorylation of ubiquitin ligase Nedd4-2. Mol Pharmacol 2014; 85: 877-886.

17. Nishikawa SI, Brodsky JL, Nakatsukasa K. Roles of molecular chaperones in endoplasmic reticulum (ER) quality control and ERassociated degradation (ERAD). J Biochem 2005; 137: 551-555.

18. Sakaguchi T, Itoh H, Ding WG, Tsuji K, Nagaoka I, Oka Y, et al. Hydroxyzine, a first generation $\mathrm{H}_{1}$-receptor antagonist, inhibits human ether-a-go-go-related gene (HERG) current and causes syncope in a patient with the HERG mutation. J Pharmacol Sci 2008; 108: $462-471$.

19. Kurata Y, Hisatome I, Matsuda H, Shibamoto T. Dynamical mechanisms of pacemaker generation in IK1-downregulated human ventricular myocytes: Insights from bifurcation analyses of a mathematical model. Biophys J 2005; 89: 2865-2887.

20. O'Hara T, Virag L, Varro A, Rudy Y. Simulation of the undiseased human cardiac ventricular action potential: Model formulation and experimental validation. PLoS Comput Biol 2011; 7: e1002061, doi:10.1371/journal.pcbi.1002061.

21. Zhao JT, Hill AP, Varghese A, Cooper AA, Swan H, LaitinenForsblom PJ, et al. Not all hERG pore domain mutations have a severe phenotype: G584S has an inactivation gating defect with mild phenotype compared to G572S, which has a dominant negative trafficking defect and a severe phenotype. J Cardiovasc Electrophysiol 2009; 20: $923-930$.

22. Wang H, Lu Y, Wang Z. Function of cardiac M3 receptors. Auton Autacoid Pharmacol 2007; 27: 1-11.

23. Kurian N, Hall CJ, Wilkinson GF, Sullivan M, Tobin AB, Willars GB. Full and partial agonists of muscarinic $\mathrm{M}_{3}$ receptors reveal single and oscillatory $\mathrm{Ca}^{2+}$ responses by $\beta_{2}$-adrenoceptors. J Pharmacol Exp Ther 2009; 330: 502-512.

24. Luo J, Busillo J, Benovic J. M3 muscarinic acetylcholine receptor- 
mediated signaling is regulated by distinct mechanisms. Mol Pharmacol 2008; 74: 338-347.

25. Guo J, Wang T, Li X, Shallow H, Yang T, Li W, et al. Cell surface expression of human ether-a-go-go-related Gene (hERG) channels is regulated by caveolin-3 protein via the ubiquitin ligase Nedd4-2. $J$ Biol Chem 2012; 287: 33132-33141.

26. Guettouche T, Boellmann F, Lane WS, Voellmy R. Analysis of phosphorylation of human heat shock factor 1 in cells experiencing a stress. BMC Biochem 2005; 6: 4.

27. Solana J, Gamberi C, Mihaylova Y, Grosswendt S, Chen C, Lasko $\mathrm{P}$, et al. The CCR4-NOT complex mediates deadenylation and degradation of stem cell mRNAs and promotes planarian stem cell differentiation. PLoS Genet 2013; 9: e1004003, doi:10.1371/journal. pgen.1004003.

\section{Supplementary Files}

Supplementary File 1

Figure S1. Family history and ECG recordings of the long QT syndrome 2 (LQT2) patient and his family members.

Figure S2. Dominant negative effects of the double mutant hERGFLAG.
Figure S3. Time-dependent effect of carbachol $(\mathrm{CCh})$ treatment duration on the maturation of WThERG-FLAG and mutant hERGFLAG (A,B).

Figure S4. Effects of carbachol on another mutant hERG, G601S hERG-FLAG, expressed in HEK293 cells.

Figure S5. Expression of M2 and M3 receptors on the HEK293 cells stably expressing WThERG-FLAG (wild-type; WT) and mutant hERG-FLAG (double mutation; DM) (A).

Figure S6. (A) Effects of the PKC inhibitor, bisindolylmaleimide (Bis-I), on WThERG-FLAG expression.

Figure S7. Simulated action potential (AP) and delayed rectifier potassium current $(\mathrm{IKr})$ behaviors of the Kurata et al (A) and O'Hara et al (B) models for human ventricular myocytes (HVMs) expressing WThERG (black) or the mutant human ether-a-go-go gene ( $h E R G)$ in the absence (red) and presence of carbachol (blue).

Figure S8. Effects of carbachol $(\mathrm{CCh})$ on expressions of the mouse ERG (mERG) and the expressed mutant hERG-FLAG in HL-1 mouse cardiomyocytes.

Please find supplementary file(s);

http://dx.doi.org/10.1253/circj.CJ-16-0712 